\title{
BIOMECHANICAL MODEL CALCULATION OF MUSCLE CONTRACTION FORCES: A DOUBLE LINEAR PROGRAMMING METHOD
}

\author{
James C. Bean and Don B. Chaffin \\ Department of Industrial and Operations Engineering, University of Michigan, Ann Arbor, MI 48109, \\ U.S.A. \\ and
}

Albert B. SCHULtZ

Department of Mechanical Engineering and Applied Mechanics, University of Michigan, Ann Arbor, MI 48109, U.S.A

\begin{abstract}
This paper presents a novel scheme for the use of linear programming to calculate muscle contraction forces in models describing musculoskeletal system biomechanics. Models of this kind are frequently found in the biomechanics literature. In most cases they involve muscle contraction force calculations that are statically indeterminate, and hence use optimization techniques to make those calculations. We present a linear programming optimization technique that solves a two-objective problem with two sequential linear programs. We use the technique bere to minimize muscle intensity and joint compression force, since those are commonly used objectives. The two linear program model has the advantages of low computation cost, ready implementation on a micro-computer, and stable solutions. We show how to solve the model analytically in simple cases. We also discuss the use of the dual problem of linear programming to gain understanding of the solution it provides.
\end{abstract}

\section{INTRODUCTION}

There is considerable interest in learning what muscle contraction forces are used to perform various physical tasks. Since these are difficult to measure in vivo, they are often calculated from biomechanical considerations. When an external moment must be equilibrated across a joint, a biomechanical model of the joint and its muscles can be established, and a set of muscle forces that equilibrate that external moment found.

However, most joints of the human body are crossed by a large number of muscles. There are at most six equations of equilibrium (three force equations and three moment equations) to be satisfied, but there are often more than six muscle forces to be calculated. For example, Seireg and Arvikar (1973) in their model of the lower extremity incorporated 29 muscles, Chao and An (1978) in their model of the hand included 18 muscles, Schultz et al. (1983) in their model of the lumbar trunk included up to 22 muscles, and An et al. (1984) in their model of the elbow included nine muscles. In such models, the calculation of the muscle contraction forces constitutes a statically indeterminate problem.

An approach often taken to solve this indeterminate problem is to use an optimization technique. For example, Seireg and Arvikar (1973) minimized sums of muscle contraction forces, Crowninshield and Brand (1981) minimized the sum of the cube of the muscle

Received May 1986; in revised form January 1987. contraction forces; An et al. (1984) minimized the muscle contraction intensity (force per unit crosssectional area); and Schultz et al. (1983) approximately minimized the muscle contraction intensity and the spine joint compression force. These optimization procedures usually provide good solutions. That is, they lead to predicted sets of muscle contraction forces that correlate well with experimentally measured levels of muscle myoelectric activity.

The techniques of Seireg and Arvikar (1973), Schultz et al. (1983) and An et al. (1984) are applications of linear programming [Hillier and Lieberman (1980), Dantzig (1963) ]. Linear programming techniques for optimization are robust enough to encompass many formulations of interest, simple enough to program on a micro-computer, and theoretically well enough developed to provide good insight into the nature of the solutions obtained.

\section{LINEAR PROGRAMMING}

A linear program is an optimization model which can be stated in the following form:

minimize $\sum_{j=1}^{n} c_{j} x_{j} \quad$ (the objective function) subject to:

$$
\begin{aligned}
\sum_{j=1}^{n} a_{i j} x_{j} & \geqslant b_{i}, i=1,2 \ldots, m \quad \text { (the constraints) } \\
x_{j} & \geqslant 0, j=1,2, \ldots, n
\end{aligned}
$$


where $c_{j}, b_{i}$ and $a_{i j}$ are known parameters and $x_{j}$ are the unknown variables. For example, in the muscle contraction force problem, the known parameters include moment arm length, line-of-action orientations, muscle cross-sectional areas, and external moments. The unknown variables are the muscle and joint reaction forces. The important feature of such models is that the objective function and the constraints must be linear in the unknowns. The constraints may be inequalities or equalities.

\subsection{Formulation of a force calculation as a linear program}

Consider a biomechanical model of a joint which requires that the three components of an external moment be equilibrated by muscles crossing the joint. Suppose we wish to minimize the muscle contraction intensity necessary to resist the external moment. The intensity is the force exerted by the muscle divided by its cross-sectional area. Let $m$ be the number of muscles modelled, $a_{i j}$ the component of the moment arm of muscle $j$ about axis $i, b_{i}$ the component of the external moment about axis $i$, and $d_{j}$ the inverse of the crosssectional area of muscle $j$. These are the known parameters of this problem. The unknowns are the muscle forces, $x_{j}$ for muscle $j$, and the resultant maximum intensity, $I$. The problem is:

\section{$\min I$}

$$
\text { subject to: } \begin{aligned}
\sum_{j=1}^{m} a_{i j} x_{j} & =b_{i}, \quad i=1,2,3 \\
d_{j} x_{j} & \leqslant I, \quad j=1,2, \ldots, m \\
x_{j} & \geqslant 0, \quad j=1,2, \ldots, m .
\end{aligned}
$$

The first set of constraints requires that the three equations of moment equilibrium be satisfied. The second set of constraints requires that the intensity of each muscle contraction be not greater than the maximum intensity, $I$. The last set requires that all muscle forces be non-negative since muscles do not sustain compression forces.

This formulation is equivalent to that used by An $e t$ al. (1984) for the elbow. This formulation provides a unique set of forces for some sets of parameters, but its solution is not unique in general, as is demonstrated in Section 3.1.

Force calculations involving other objectives can be formulated as linear programs. For example, if the objective were to minimize the sum of muscle contraction forces, given a fixed maximum intensity, the model would be:

$$
\begin{gathered}
\min \sum_{j=1}^{m} x_{j} \\
\text { subject to: } \sum_{j=1}^{m} a_{i j} x_{j}=b_{i}, \quad i=1,2,3 \\
d_{j} x_{j} \leqslant I, \quad j=1,2, \ldots, m \\
x_{j} \geqslant 0, \quad j=1,2, \ldots m
\end{gathered}
$$

where $I$ is now considered fixed and only the $x_{i}$ are the unknowns.

The following discussion of solution techniques and sensitivity is valid for any problem that can be formulated as a linear program.

\subsection{Solution of the linear program}

The method most commonly used to find the optimal solution to a linear program is Dantzig's simplex algorithm. A feasible solution is defined as any solution that satisfies all the constraints, regardless of optimality. The set of feasible solutions to any linear program can be shown to be a polyhedron such as that in Fig. 1. Since muscles can produce finite maximum forces, the feasible region is bounded (a polytope). Hence, it is known that an optimal solution must lie at one of the corners of the feasible region. The simplex method moves from one corner to the next until the best solution is found. While the number of corners can be large, this technique is efficient and moves quickly to the optimal corner.

Many software packages are available for both mainframe and micro-computers to solve linear programs. The computational results presented later in this paper were run on a mainframe using a package called LPSUB [Davisson (1969)] and on an IBM PCXT using LP.BAS and the MATRIX-100 enhancement for BASIC [both by Stanford Business Software (1984)].

To illustrate these ideas, consider a hypothetical two-muscle model of the lumbar trunk required to equilibrate one moment. Suppose the muscle moment arm lengths are $6.5 \mathrm{~cm}$ and $1.0 \mathrm{~cm}$ and the muscle cross-sectional areas are $10 \mathrm{~cm}^{2}$ and $100 \mathrm{~cm}^{2}$, respectively. Areas this large are chosen since the model muscles would represent several smaller muscles. Suppose that the external moment is $58 \mathrm{Nm}$ and that contraction intensity is not to exceed $40 \mathrm{~N} / \mathrm{cm}^{2}$. To minimize the total force exerted by the two muscles requires:

$$
\min x_{1}+x_{2}
$$

subject to: $0.065 x_{1}+0.01 x_{2}=58$

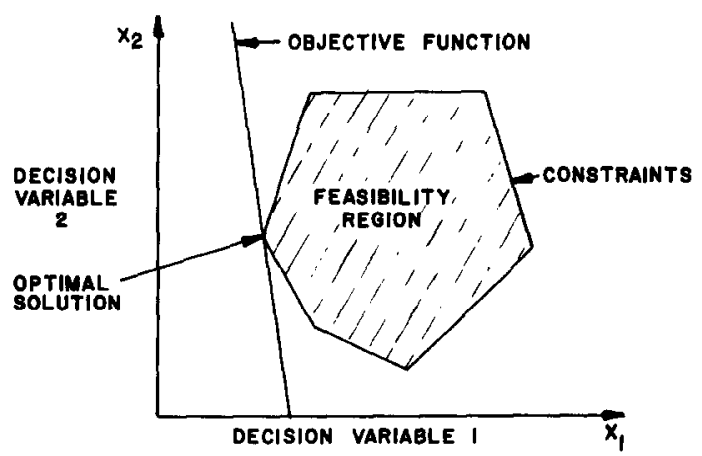

Fig. 1. Typical problem having two unknowns with objective shown for minimum values that are feasible. 


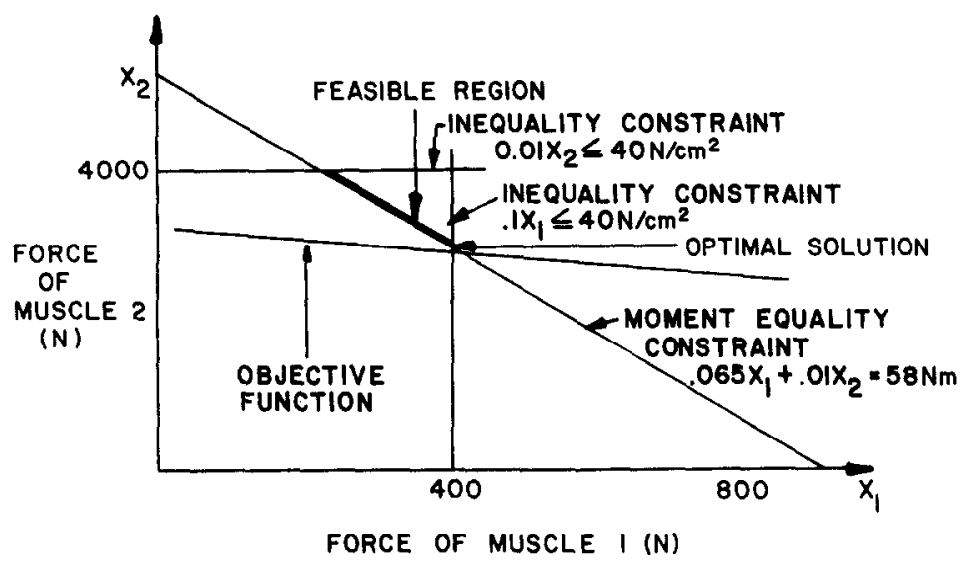

Fig. 2. Two muscle examples with two inequality and one equality constraints.

$$
\begin{gathered}
0.1 x_{1} \leqslant 40,0.01 x_{2} \leqslant 40 \\
x_{1} \geqslant 0, x_{2} \geqslant 0 .
\end{gathered}
$$

Figure 2 shows the polyhedron formed by the feasible region. In this example the feasible region collapses to a line segment. The optimal solution must lie at one of its two endpoints. The objective function has values of $4277 \mathrm{~N}$ at the left endpoint and $3600 \mathrm{~N}$ at the right. The optimal solution is at the right endpoint, where $x_{1}=400 \mathrm{~N}, x_{2}=3200 \mathrm{~N}$.

If maximum intensity is to be minimized, the model changes to:

$$
\begin{gathered}
\min \mathrm{I} \\
\text { subject to: } 0.065 x_{1}+0.01 x_{2}^{\prime}=58 \\
0.1 x_{1} \leqslant I, 0.01 x_{2} \leqslant I \\
x_{1} \geqslant 0, x_{2} \geqslant 0 .
\end{gathered}
$$

$\left(\mathrm{N} / \mathrm{cm}^{2}\right)$

The solution to this is $x_{1}=351.52 \mathrm{~N}, x_{2}=3515.15 \mathrm{~N}$, and $I=35.15 \mathrm{~N} / \mathrm{cm}^{2}$. This solution has a lower maximum intensity, but higher total muscle force, which results in higher joint compression force. These examples, show the trade-off between the objectives of low contraction intensity and low joint compression force. The theory of linear programming allows us to evaluate this trade-off.

\subsection{Sensitivity of solutions}

Associated with every linear program is another linear program called its dual problem. In general, the dual has one constraint for each unknown in the original problem, and one unknown for each constraint in the original problem. Hillier and Lieberman (1980) and Dantzig (1963) discuss duality in linear programming in depth. We will discuss only the optimal solution to the dual problem and its provision of information about sensitivity of the solution in the original problem. We will illustrate this with our example of the intensity vs compression trade-off.
While the optimal solution to a linear program gives the contribution to the objective function of each variable (the muscle forces in our example), the solution to the dual problem gives the contribution of each constraint to the same objective. In our example, the dual solution shows how much of the total muscle force arises because of the external force, and how much because of the upper bounds on intensity. In other words, it describes how the objective value would change if the $b_{i}$ and $I$ values were changed.

The optimal solution to the dual of a linear program has the economic interpretation of an internal price for an additional unit of some scarce resource. In the linear program formulated above the prices for the moment equation tell how much intensity would change if an additional $\mathrm{Nm}$ of external moment were applied. This concept will be demonstrated below. Most computer packages to solve linear programs report the prices when the problem is solved. It is not necessary to solve the related dual linear program; this information is available directly from the solution to the original linear program.

\subsection{Example: four muscle, two moment model of the} lumbar trunk

Consider a model of the lumbar trunk at the L5/S1 level that incorporates four single-equivalent muscles, the erector spinae, latissimus dorsi, oblique abdominals, and the psoas (Fig. 3). Suppose the moment arms for the erector spinae and latissimus are $7.4 \mathrm{~cm}$ and $9.8 \mathrm{~cm}$, respectively, and these muscles lie in the mid. sagittal plane. The oblique and psoas muscles have moment arms of $14.7 \mathrm{~cm}$ and $6.0 \mathrm{~cm}$, respectively, and lie in the coronal plane passing through the center of the vertebral body. Further suppose the external moment is $58 \mathrm{Nm}$ in flexion, and $41 \mathrm{Nm}$ in lateral bending. This would correspond to holding approximately a $200 \mathrm{~N}$ weight at a distance of $35 \mathrm{~cm}$ from L5/S1 disc. Take muscle cross-sectional areas as. respectively, $31,3,13,5 \mathrm{~cm}^{2}$. The linear program to minimize the maximum muscle contraction intensity 


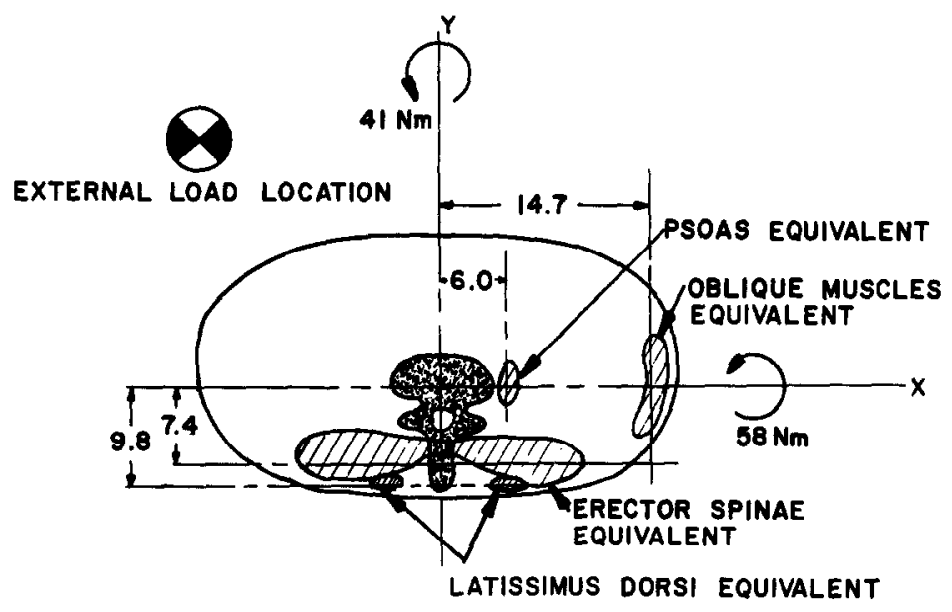

Fig. 3. Hypothetical four muscle torso model with moment arms and cross-sectional areas selected as suggested by Schultz et al., 1983, Reid and Costigan, 1985 and Nemeth and Ohlsen, 1986. The muscle lines of action have been located so that the erector and latissimus dorsi muscles act to only resist $X$-axis moment, and psoas and oblique muscles act to only resist $Y$-axis moment created by $200 \mathrm{~N}$ load acting $35.5 \mathrm{~cm}$ from L5/S1 disc.

to equilibrate the moments is then:

$\min I$

subject to: $0.068 x_{1}+0.091 x_{2}=58$

$$
0.073 x_{3}+0.023 x_{4}=41
$$$$
0.03226 x_{1} \leqslant I, \quad 0.33333 x_{2} \leqslant I
$$$$
0.07692 x_{3} \leqslant I, \quad 0.2 x_{4} \leqslant I
$$$$
x_{j} \geqslant 0, j=1,2,3,4 \text {. }
$$

$\left(\mathrm{N} / \mathrm{cm}^{2}\right)$

An optimal solution yields muscle forces of erector spinae $=698 \mathrm{~N}$, latissimus dorsi $=116 \mathrm{~N}$, abdominal oblique $=501 \mathrm{~N}$, and psoas $=193 \mathrm{~N}$, respectively, and requires a maximum intensity of $38.5 \mathrm{~N} / \mathrm{cm}^{2}$. The intensity for each individual muscle is $22.5,38.5,38.5$, $38.5 \mathrm{~N} / \mathrm{cm}^{2}$, respectively.

The dual solution gives us six sensitivities for this example: one for each moment equation and one for each intensity bound. Their values, are $0 \mathrm{Nm}, 0.94 \mathrm{Nm}$, $0 \mathrm{~N} / \mathrm{cm}^{2}, 0 \mathrm{~N} / \mathrm{cm}^{2},-0.89 \mathrm{~N} / \mathrm{cm}^{2},-0.11 \mathrm{~N} / \mathrm{cm}^{2}$. The 0.94 signifies that if the external moment in the lateral plane were increased from 41 to $42 \mathrm{Nm}$, the required maximum intensity would increase $0.94 \mathrm{~N} / \mathrm{cm}^{2}$ from $38.5 \mathrm{~N} / \mathrm{cm}^{2}$ to $39.4 \mathrm{~N} / \mathrm{cm}^{2}$. However, if the moment in the sagittal plane were increased, no change in the maximum intensity would be necessary. This occurs because of the slack in the intensity of the erector spinae contraction $\left(22.5\right.$ required vs $38.5 \mathrm{~N} / \mathrm{cm}^{2}$ allowed).

\section{A DOUBLE LINEAR PROGRAMMING METHOD}

\subsection{Background}

We propose a new method for calculation of muscle contraction forces using biomechanical models of the musculoskeletal system. We will describe that method through an illustration in the four muscle, two moment model of the lumbar trunk, although its applicability is more general than this.

Schultz et al. (1983) described lumbar trunk models that minimize the compression on the spine while, at the same time, minimizing maximum muscle contraction intensity. Such models provide results that are in better agreement with experimental measurements than those provided by less sophisticated models. The method we propose efficiently meets such double objectives.

Compression on the spine is a function of the muscle contraction forces, body segment weights, external forces and moments, and perhaps intra-abdominal pressure. Usually, the only quantities in this list that are not prescribed are the muscle contraction forces. The objective to minimize spine compression is equivalent to the linear program described by Relations (2) listed in Section 2.1.

The bounds on muscle intensity arise from our objective to minimize maximum contraction intensity. If these bounds are too low the resulting model will be infeasible. That is, there will be no combination of muscle forces, bounded by that intensity, that can equilibrate the external loads. If the intensity value is too high, muscles with larger moment arms will be recruited before those with smaller ones, contrary to what is observed experimentally.

Schultz et al. (1983) solved this problem by solving a sequence of linear programs of the type described by Relations (2), each with a different upper bound for intensity selected by a step-wise procedure, as follows. They began with low intensities which lead to infeasible models, and gradually increased $I$ until the first feasible solution was found. The difficulties of this approach are its substantial computational require- 
ments and instability of solutions as intensity values are changed slightly. The instability results when an optimal solution occurs at one corner of the feasible polyhedron for a given $I$ value, but jumps to another corner with a very small change in $I$.

We propose an alternative to this procedure which involves formulating and solving two linear programs sequentially. First, as in An et al. (1984b), minimize maximum muscle intensity using Relations (1). Let the optimal intensity value from this solution be $I^{*}$. Second, solve the linear program using Relations (2) to minimize the sum of the muscle forces, using $I^{*}$ as the intensity limit. The first linear program determines the lowest intensity value which allows feasible solutions. The second linear program chooses amongst these solutions to minimize spinal compression. Hence, this scheme addresses both of the stated objectives.

This approach gives results which differ from those of previously published techniques. To demonstrate this, consider the four muscle, two moment model introduced in Section 2.4 (Fig. 3). Results using four approaches are compared in Table 1. The approaches are (1) minimize compression with no constraint on maximum muscle intensity, (2) minimize largest necessary intensity, (3) minimize both through a sequence of solutions, and (4) minimize both through the two linear program approach just outlined. Approaches (1), (3), and (4) determine unique solutions. Approach (2) may have many optimal solutions. Since there are multiple optima, the particular optimal solution chosen by the code will depend on the ordering of the unknowns and constraints, and tie-breaking conventions in the code. Three optimal solutions are listed in the table denoted by ' $2 a$ ', ' $2 b$ ', and ' $2 c$ '. Note that the ignored compression objective changes between the three optimal solutions.

In this simple case, if muscle intensity is not constrained while seeking to minimize compression (Model 1), the maximum stabilizing forces will be allocated to the muscles with the largest effective moment arms (latissimus dorsi and abdominal oblique muscles). This results in a lower compression force on the spine but muscles are not necessarily recruited as observed in vivo and will be highly stressed.

Table 1. Solutions using various approaches

\begin{tabular}{llcccrrr}
\hline $\begin{array}{c}\text { Model } \\
\text { objectives: } \\
\text { minimize }\end{array}$ & $\begin{array}{c}\text { Erector } \\
\text { spinae } \\
(\mathrm{N})\end{array}$ & $\begin{array}{c}\text { Latissimus } \\
\text { dorsi } \\
(\mathrm{N})\end{array}$ & $\begin{array}{c}\text { Abdominal } \\
\text { oblique } \\
(\mathrm{N})\end{array}$ & $\begin{array}{r}\text { Psoas } \\
(\mathrm{N})\end{array}$ & $\begin{array}{c}\text { Necessary } \\
\text { intensity } \\
\left(\mathrm{N} / \mathrm{cm}^{2}\right)\end{array}$ & $\begin{array}{c}\text { Spinal } \\
\text { comp. } \\
(\mathrm{N})\end{array}$ \\
\hline 1 & Spinal comp. & 0.0 & 591.8 & 278.9 & 0.0 & 197.3 & 870.7 \\
2a & Nec. int. & 694.7 & 67.2 & 278.9 & 0.0 & 22.4 & 1040.8 \\
2b & Nec. int. & 694.7 & 67.2 & 241.1 & 92.7 & 22.4 & 1095.7 \\
2c & Nec. int. & 694.7 & 67.2 & 233.2 & 112.1 & 22.4 & 1107.2 \\
3 & Sequential LP & 692.4 & 69.0 & 278.9 & 0.0 & 23.0 & 1040.3 \\
4 & Double LP & 694.7 & 67.2 & 278.9 & 0.0 & 22.4 & 1040.8 \\
\hline
\end{tabular}

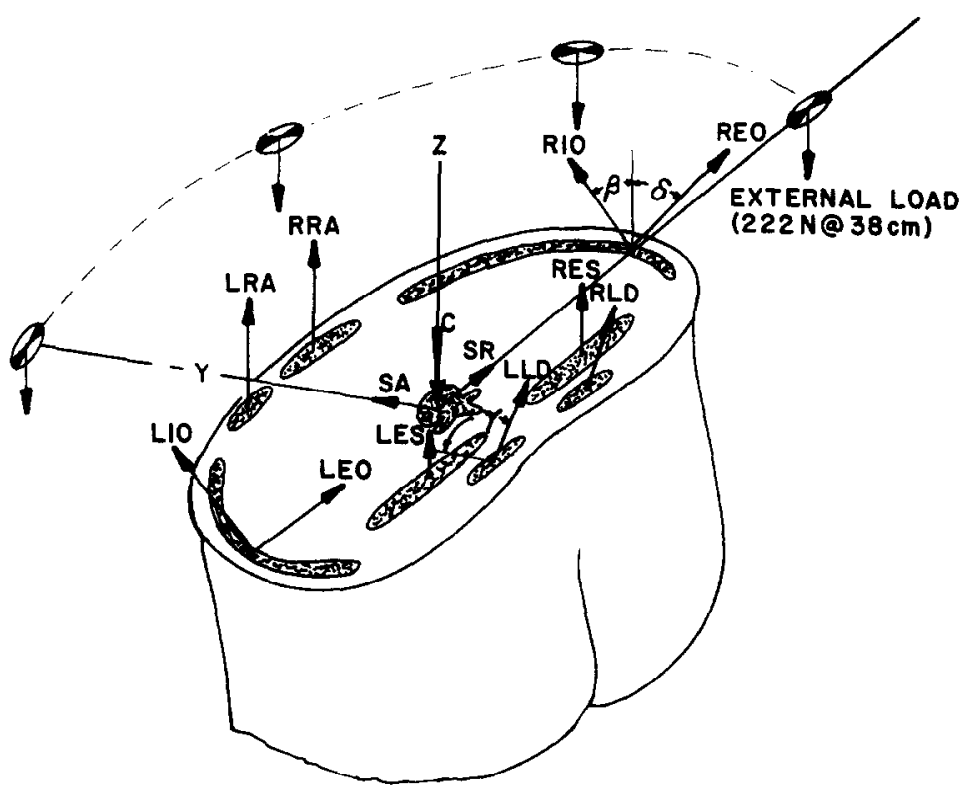

Fig. 4. Schematic diagram of the 10 muscle model. The 10 unknown muscle forces are computed to predict the minimum compression force $\mathrm{C}$ on the disc using the new multi-objective, double linear program method, (adapted from Schultz et al., 1982a). 


\subsection{Solution sensitivity}

In addition to the advantages of low computation cost and stability of solutions, an advantage of the double linear programming approach is that it provides the sensitivities which can be used in our example to calculate the trade-off between spine compression and muscle intensity. The intensity determined in the first linear program, $I^{*}$, becomes a constant in the second linear program, forming there the right hand side of the intensity upper bound for each unknown force. When the second linear program is solved, sensitivities are determined for each of these constraints. The sensitivity for constraint $d_{j} x_{j} \leqslant I^{*}$ is the change in compression that would result from that constraint if the intensity were increased by one $\mathrm{N} / \mathrm{cm}^{2}$. Since the same value of $I^{*}$ is found in each such constraint, the total effect is the sum of the sensitivities for each intensity upper bound.

In the four muscle, two moment model, the sensitivities from the second linear program were 13.5, 6.8, $0,-1.0,0$, and $0 \mathrm{~cm}^{2}$, the first two corresponding to the moment equilibrium equations, and the last four corresponding to the intensity upper bounds. The sum of the sensitivities corresponding to the intensity bounds is $-1.0 \mathrm{~cm}^{2}$. Hence, if intensity is allowed to increase one $\mathrm{N} / \mathrm{cm}^{2}$ from 22.4 to 23.4 , spine compression would decrease $1.0 \mathrm{~cm}^{2} \times 1 \mathrm{~N} / \mathrm{cm}^{2}=1.0 \mathrm{~N}$.

The sensitivities can also be used to determine the change in spine compression per unit change in external moment in some direction. In this case we have two countering effects. A larger moment requires larger muscle forces, and hence spine compression, to counter it. However, with higher forces, the necessary intensity previously is no longer valid. A larger value will be necessary and, as seen above, leads to reduced compression.

Consider an increase of $1 \mathrm{Nm}$ of moment in the sagittal plane. Let $\pi_{s}^{1}$ and $\pi_{s}^{2}$ be the sensitivities of the lateral moment constraint in the first and second linear programs, respectively. Let $\pi_{I}^{2}$ be the sum of the prices for the four intensity upper bounds in linear program two. Then the net change in compression due to a unit increase in moment in the sagittal plane is

$$
\pi_{s}^{2}+\pi_{I}^{2} \pi_{s}^{1}
$$

In our example this value is $13.1 \mathrm{~N}$. An additional effect is that minimum intensity increases $0.39 \mathrm{~N} / \mathrm{cm}^{2}$ $\left(=\pi_{s}^{1}\right)$.

\subsection{Single moment models}

If only one moment equation appears in the model, the two linear program model is equivalent to minimizing the maximum intensity. In this case the solution to the first linear program is known to have a unique solution. Hence, the second linear program has only one solution to choose from. Moreover, it is not necessary to solve any linear programs. The optimal solution can be determined analytically.

The muscles in this model can be separated into two groups: those working to counter the external moment (agonists), and those adding to the external moment (antagonists). As in Chaffin and Park (1973), we know that the antagonistic muscle forces can be set to zero without loss of optimality. If any antagonistic muscle acted, some agonist muscle would be forced to higher intensity to counter this antagonistic effect, resulting in a potentially inferior solution. This assumption cannot be made if more than one external moment is present since the 'antagonistic' muscle may be an agonist in resisting the other moment.

We can also show that maximum intensity is minimized when all agonist muscles have equal intensity. Given any solution where this was not the case, redistributing some of the load from the muscle contracting with highest intensity to muscles with lower intensities would decrease maximum intensity. Then for each muscle, $j$, the intensity $d_{j} x_{j}$ equals $I$ for some intensity value uniform over the muscles. Hence, $x_{j}=I / d_{j}$. Substitute this into the moment equation to get

$$
\sum_{j=1}^{n} a_{j}\left(I / d_{j}\right)=b=I \sum_{j=1}^{n}\left(a_{j} / d_{j}\right) .
$$

Hence, $I=b /\left[\sum_{j=1}^{n .}\left(a_{j} / d_{j}\right)\right]$ and $x_{j}=I / d_{j}$.

For example, consider the two muscle, single moment example in Section 2.2. Then

$$
\begin{aligned}
I=\frac{b}{\sum_{j=1}^{n}\left(a_{j} / d_{j}\right)} & =\frac{58}{0.065 / 0.1+0.01 / 0.01} \\
& =35.15 \mathrm{~N} / \mathrm{cm}^{2},
\end{aligned}
$$

and

$$
x_{1}=I / 0.1=351.52 \mathrm{~N}
$$

and

$$
x_{2}=I / 0.01=3515.15 \mathrm{~N} .
$$

This analytical technique results in the same solution found by the linear program in Section 2.2.

We have not made any assumption to simplify the problem to a determinate one, but rather, have analytically found the optimal solution to the indeterminate system.

\section{COMPUTATIONAL TESTING}

Linear programming provides a powerful method of studying complex muscular actions. The double linear programming method presented here seems superior to linear programming schemes used earlier. We will further illustrate its application using 10 muscle model of the lumbar trunk developed by Schultz et al. (1982) (Fig. 4). In all calculations, a linear program is solved first to find the lowest maximum muscle intensities necessary to resist the external moment applied to the L5/S1 trunk cross-section and second, using this intensity bound, to select the muscle actions which minimize the compression force on the $\mathrm{L} 5 / \mathrm{S} 1 \mathrm{disc}$. Note that data used for these tests are not related to the example data from Sections 2 and 3. 


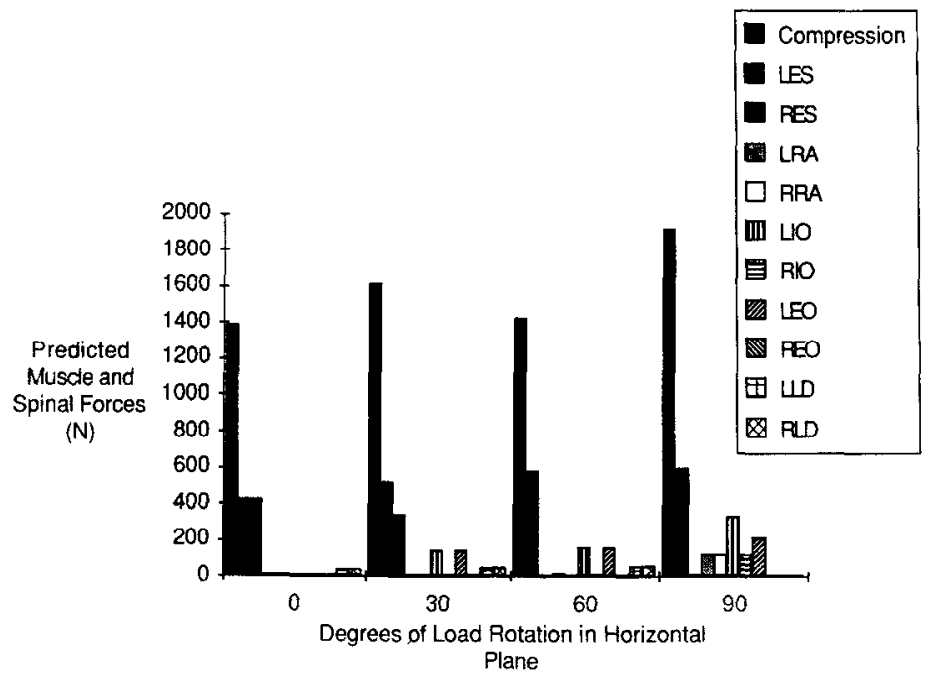

Fig. 5. Predicted muscle contraction and L5/S1 compression forces with $222 \mathrm{~N}$ load acting at $38 \mathrm{~cm}$ from disc using multi-objective, double linear program approach.

External moments were systematically varied from a pure sagittal plane moment to a pure coronal or frontal plane (lateral bending) moment. We assumed an average-sized male was standing erect and holding a weight of $222 \mathrm{~N}$. This weight was shifted in the horizontal plane from directly in front of the body to the right side, on a circular arc of radius $38 \mathrm{~cm}$ from the $\mathrm{L} 5 / \mathrm{S} 1$ disc center. Muscle forces were computed at each $30^{\circ}$ of angular displacement of the weight.

From the calculated muscle contraction and spine compression forces (Fig. 5) we conclude:

(1) When the external moment is purely a flexion moment, only the erector spinae muscles (LES and RES) are active. These agonist contractions account for approximately $60 \%$ of the spine compression force, with the components of body weight and weight held in the hand accounting for the remainder.

(2) When the held weight is shifted as little as $30^{\circ}$ to the right of the mid-sagittal plane, the left internal (LIO) and external oblique (LEO) muscles become active, while the right erector spinae (RES) contract less than at $0^{\circ}$.

(3) At $60^{\circ}$ of rotation from the mid-sagittal plane, the left oblique and left erector spinae muscles can equilibrate the external bending moment, and the right erector spinae are not needed.

(4) As the weight is rotated $90^{\circ}$ from mid-sagittal plane (i.e. a pure right lateral bending moment is induced) both left oblique muscles become quite active. Also some rectus abdominus activity and right internal oblique activity become noticeable. Such behavior has been documented by Schultz et al. (1982) with myoelectric measurements. The right oblique and rectus abdominus muscles are acting to stabilize the column against the complex effects of the left obliques. Because of this complex recruitment pattern, the overall compression force is highest when the bending moment is purely lateral direction, as opposed to purely sagittal plane flexion.

\section{SUMMARY}

The use of double objective linear programming methods provides a means to study musculoskeletal biomechanics. Systematic investigations of these mechanics have been impeded in the past by the magnitudes of the computational efforts required. The double linear programming method is particularly attractive because efficient linear programming codes are now available on personal computers.

We have used models of the low-back to illustrate how our method can be used to solve complex biomechanical problems. Our scheme can be formulated in many different ways to solve particular problems. We have shown how use of the solution to the dual problem provides insight into solution sensitivity.

The methods presented provide the means to formulate and solve more complex biomechanical problems than previously practical. Attention can now more readily be concentrated on comparisons of model predictions with measured human performance data.

Acknowledgements - We would like to thank George Page, Richard Hughes and Carl Luchies for executing the computational studies described in this paper. We thank Gunnar Andersson for his helpful comments on preliminary findings from the research. The work of A. B. Schultz was supported in part by U.S. Public Health Service Grants OHO1962 and NS20536. The work of D. B. Chaffin was partially supported by research funding from Ford, Owens Corning Fiberglass, and GenCorp Corporations.

\section{REFERENCES}

An, K. N., Kwak, B. M., Chao, E. Y. and Morrey, B. F. (1984) Determination of muscle and joint forces: a new technique to solve the indeterminate problem. J. biomech. Engng 106, 364-367. 
Chaffin; D. B. and Park, K. S. (1973) A longitudinal study of low-back pain as associated with occupational weight lifting factors. Am. ind. Hyg. Ass. J. 34, 513-525.

Chao, E. and An, K. (1978) Graphical interpretation of the solution to the redundant problem in biomechanics. J. biomech. Engng 100, 159-167.

Crowninshield, R. and Brand, R. (1981) A physiologically based criterion of muscle force prediction in locomotion. J. Biomechanics 14, 793-801.

Dantzig, G. B. (1963) Linear Programming and Extensions. Princeton University Press, Princeton.

Davisson, C. (1969) LPSUB Linear Programming Package, Naval Research Laboratory, Washington, DC.

Hillier, F. and Lieberman, G. (1980) Operations Research, third edn. Holden-Day, San Francisco.

Nemeth, G. and Ohlson, H. (1986) Moment arm lengths of trunk muscles to the lumbosacral joint obtained in vivo with computer tomography. Spine 2, 158-160.

Reid, J. G. and Costigan, P. A. (1985) Geometry of adult rectus abdominus and erector spinae muscles. J. Orthop. Sports Physical Therapy 6, 278-280.

Schultz, A. B., Andersson, G. B. J., Haderspeck, K., Örtengren, R., Nordin, M. and Björk, R. (1982) Analysis and measurement of lumbar trunk loads in tasks involving bends and twists. J. Biomechanics 15, 669-675.

Schultz, A. B., Haderspeck, K., Warwick, D. and Portillo, D (1983) Use of lumbar trunk muscles in isometric perform ance of mechanically complex standing tasks. J. orthop Res. $1,77-91$.

Seireg, A. and Arvikar, R. (1973) A mathematical model for evaluation of forces in lower extremities of the muscular system. J. Biomechanics 6, 313-326.

Stanford Business Software, Inc. (1984) Matrix-100. 2672 Bayshore Parkway, Suite 304, Mountain View, CA, 94043. 